CALIDAD EN EL SERVICIO ELECTRÓNICO EN UNA REPOSTERÍA DE LA CIUDAD DE MÉRIDA, YUCATÁN, MÉXICO.

\title{
CALIDAD EN EL SERVICIO ELECTRÓNICO EN UNA REPOSTERÍA DE LA CIUDAD DE MÉRIDA, YUCATÁN, MÉXICO
}

\section{QUALITY OF THE ELECTRONIC SERVICE IN A PASTRY SHOP IN THE CITY OF MÉRIDA, YUCATÁN, MÉXICO}

\author{
Francisco Iván Hernández Cuevas*, Fredi Israel Mex Arriaga**,

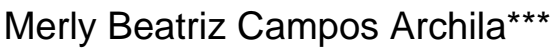

* Doctor en Ciencias Sociales, Profesor de Asignatura. Universidad Tecnológica Metropolitana. ORCID: http://orcid.org/0000-0002-1310-7574.

** Maestro en Planificación de Empresas y Desarrollo Regional, Profesor de Asignatura. Universidad Tecnológica Metropolitana. Email: fredi.mx@pa.metropolitana.edu.mx, ORCID: http://orcid.org/0000-00025727-3347.

*** Licenciada en Gastronomía, Egresada de la Universidad Tecnológica Metropolitana. Email: 16090127@alumno.utmetropolitana.edu.mx, ORCID: http://orcid.org/0000-0003-4972-0116.

Dirección para recibir correspondencia: francisco.hernandez@pa.utmetropolitana.edu.mx 
CALIDAD EN EL SERVICIO ELECTRÓNICO EN UNA REPOSTERÍA DE LA CIUDAD DE MÉRIDA, YUCATÁN, MÉXICO.

\section{RESUMEN}

El artículo presenta una propuesta de escala de medición para la calidad en el servicio electrónico en microempresas del ámbito repostero con pruebas de confiabilidad y estima los resultados de éste para una repostería de la ciudad de Mérida, Yucatán, México.

OBJETIVO: Proponer y estimar una escala para la medición de la calidad en el servicio electrónico para microempresas del sector de preparación de alimentos y bebidas, particularmente en el ámbito de repostería.

MATERIAL Y MÉTODO: Se realizó un estudio con enfoque cuantitativo, de tipo no experimental, de alcance descriptivo y de corte transversal, tomando como referencia los trabajos de Stevens, Knuston y Patton (1995) para la propuesta de la escala de medición. Se utilizaron encuestas electrónicas que fueron almacenadas en una hoja de cálculo para su posterior análisis en STATA.

RESULTADOS: El instrumento propuesto resultó con una confiabilidad alta (0.94) y con un resultado global promedio de 4.84 puntos de 5.0 posibles, donde las dimensiones más altas resultaron ser elementos tangibles (4.89), calidad de los alimentos y empatía (ambos con 4.88 cada uno). Las dimensiones con menor puntuación registrada fueron la atención electrónica al cliente (4.79) y confiabilidad (4.78).

CONCLUSIONES: Se hace constar la utilidad de la escala propuesta para microempresas reposteras que han comenzado a digitalizar sus servicios, obteniendo niveles de confiabilidad bastante altos. Ante este escenario, es necesario generar mayores instrumentos desde la ciencia administrativa que permitan un mayor grado de profesionalización de las empresas, así como también una ruta para una digitalización más precisa.

PALABRAS CLAVE: Calidad en el servicio. Restaurante. Atención al cliente. Satisfacción. 
CALIDAD EN EL SERVICIO ELECTRÓNICO EN UNA REPOSTERÍA DE LA CIUDAD DE MÉRIDA, YUCATÁN, MÉXICO.

\section{ABSTRACT}

This article presents a proposal for a measurement scale for the quality of the electronic service in micro-enterprises in the pastry field with reliability tests and also estimates its results for a pastry store in the city of Mérida, Yucatán, Mexico.

OBJECTIVE: To propose and estimate a scale to measure the quality of electronic services for micro-enterprises in the food and beverage preparation sector, particularly in the pastry field.

MATERIAL AND METHOD: For the proposal of the measurement scale, a study with a quantitative approach, non-experimental, cross-sectional and descriptive in scope was carried out, and the works of Stevens, Knuston and Patton (1995) were taken as reference. Electronic surveys were used, and stored in a spreadsheet for later analysis in STATA.

RESULTS: The proposed instrument had a high reliability (0.94) and an average global result of 4.84 points out of 5.0 , where the highest dimensions were tangible elements (4.89), food quality and Empathy (each one with 4.88). The dimensions with the lowest score recorded were electronic customer service (4.79) and reliability (4.78).

CONCLUSIONS: The usefulness of the proposed scale for micro-pastry companies that have begun to digitize their services is noted, obtaining fairly high levels of reliability. Faced with this scenario, it is necessary to generate more instruments from the administrative science perspective which allow a greater degree of professionalization of companies, as well as a route for a more accurate digitization.

KEYWORDS: Quality of service. Restaurant. Customer service. Satisfaction. 
CALIDAD EN EL SERVICIO ELECTRÓNICO EN UNA REPOSTERÍA DE LA CIUDAD DE MÉRIDA, YUCATÁN, MÉXICO.

\section{INTRODUCCIÓN}

La creciente evolución tanto en las expectativas como en la capacidad de percepción de los consumidores sobre los productos y servicios que demandan se ha derivado de diversos factores como: el entorno social, mayor y mejor educación, procesos competitivos potenciados por la globalización, desarrollo tecnológico, influencias culturales, entre otros (Guzmán y Cárcamo, 2014). Por lo tanto, el análisis de la calidad del servicio ha adquirido una gran importancia en todos los negocios, ya que las empresas que desean destacar o mantenerse dentro del mercado, deben ofrecer productos y servicios de calidad para poder responder a una amplia serie de necesidades en el mercado (Hernández, et al., 2019).

Este comportamiento no es ajeno a las industrias que están relacionadas con la preparación de alimentos y bebidas, los cuales se han visto en muchas ocasiones forzados a innovar para adaptarse a las nuevas demandas y estándares que exige la sociedad, en definitiva, la calidad en el servicio con el paso del tiempo se ha vuelto más relevante en los negocios dedicados a la elaboración de alimentos y bebidas. El poder realizar un profundo análisis sobre la calidad en el servicio es una gran ventaja, debido a que los resultados del estudio tienen la capacidad de fungir tanto como herramienta de diagnóstico, como de generación de estrategias centradas en el establecimiento y que tienen como fin aumentar la satisfacción del cliente al consolidar su lealtad, a la par de la creciente capacidad de generación de nueva clientela (Guzmán y Cárcamo, 2014) que al final se convierten en un potencial incremento de los ingresos y la disminución de costos operativos.

Es importante destacar, que la calidad del servicio varía aún en las mismas actividades económicas, es decir, cada tipo de industria posee características particulares que van desde el aspecto del producto, la experiencia recibida y las expectativas que tienen los clientes (Bolton y Drew, 1994).

Ante la contingencia sanitaria ocasionada por el Covid-19, grandes sectores de la economía tanto internacional, nacional y local han tenido que disminuir o pausar sus actividades por completo. Para mitigar esta situación, muchas empresas sobre todo en el ámbito restaurantero han incursionado y otras han intensificado el uso de medios digitales para poder seguir ofreciendo un servicio a distancia, llevando la experiencia física a un entorno más digital que en la mayoría de 
CALIDAD EN EL SERVICIO ELECTRÓNICO EN UNA REPOSTERÍA DE LA CIUDAD DE MÉRIDA, YUCATÁN, MÉXICO.

los casos se ha manifestado en el uso de plataformas digitales de entrega de servicios a domicilio, el uso de redes sociales para atención y venta, o el diseño completo de una página web.

Esta forma de prestar un servicio sigue siendo aceptada en el contexto actual del uso de Internet, ya que, para el análisis de la calidad en un servicio electrónico, es indispensable tomar en cuenta todos aquellos sucesos que pueden ocurrir antes, durante y después de las transacciones (Zeithaml, Parasuraman y Malhotra, 2002).

En este orden de ideas, Barrero, Navarro y Peris (2014) diferencian dos tipos de interacciones con el uso de la internet y las transacciones económicas: 1) prestación de servicio sin interacciones presenciales, donde los clientes son quienes reciben el servicio por sí mismos, sin la presencia de ningún representante del negocio, y 2) prestación de servicios con interacciones presenciales y no presenciales, que ocurren cuando un cliente requiere solucionar cualquier conflicto o duda que pueda surgir durante la prestación del servicio, o cuando la empresa no ha automatizado su interacción en línea.

No cabe duda, que cuando una empresa comienza su digitalización, son múltiples los beneficios potenciales a los cuales se puede hacer acreedora, González y Martínez (2014) argumentan que se pueden eliminar actividades monótonas, reasignar puestos con mayor valor agregado (actividades que necesiten un nivel más alto de cualificación), generar más información de manera digital que permita la realimentación inmediata y específica con la finalidad de mejorar la eficiencia de los procesos físicos, la mejora potencial de la calidad de los productos y servicios ofertados, entre otros más, pero los autores coinciden que el verdadero potencial se encuentra en la posibilidad de redefinir el núcleo de la empresa y cambiar el entorno que la delimita.

El nivel de digitalización que presente una empresa es uno de los elementos vitales para poder evaluar la calidad en el servicio electrónico proporcionado. La literatura ha examinado en numerosos estudios este fenómeno, por una parte, al tratar de aplicar las dimensiones de un servicio tradicional de la famosa escala ServQual (Kanyama y Black, 2000) o también proponiendo nuevas dimensiones como Wolfinbarger y Gilly (2003).

Como el presente documento se centra en la industria restaurantera, particularmente en una repostería local de la ciudad de Mérida, Yucatán, la cual por medio la aplicación de herramientas digitales como Facebook e Instagram han logrado llegar a más clientes y mantener sus procesos 
CALIDAD EN EL SERVICIO ELECTRÓNICO EN UNA REPOSTERÍA DE LA CIUDAD DE MÉRIDA, YUCATÁN, MÉXICO.

productivos; es decir, se encuentra en una fase inicial en la digitalización empresarial, se decidió proponer una nueva escala basada en la propuesta por Hernández, et al. (2017), la cual se inspira en el modelo DINESERV de Stevens, Knuston y Patton (1995), a la cual se le añadió una dimensión relacionada con la atención electrónica.

En total, el modelo propuesto tiene siete dimensiones, las cuales son descritas a continuación:

Tabla 1

Dimensiones del modelo propuesto

\begin{tabular}{ll}
\hline Dimensión & \multicolumn{1}{c}{ Definición } \\
\hline Atención electrónica & $\begin{array}{l}\text { Centrada en las interacciones del establecimiento a través de } \\
\text { medios electrónicos, así como la comunicación e información } \\
\text { compartida. }\end{array}$ \\
Elementos tangibles & Hace referencia a la infraestructura física y tecnológica, equipo, \\
& personal y material de comunicación. \\
Confiabilidad & Se refiere a todo lo relacionado con el estado físico del proceso de \\
& compraventa de los alimentos: etiquetado, la facturación precisa y \\
& recibir, específicamente, lo ordenado por el cliente. \\
Capacidad de respuesta & Hace referencia la interacción con el personal, con la carta- \\
& menú de alimentos y/o bebidas o cuando le brinda asistencia; \\
la respuesta adecuada y rápida respecto a las necesidades y \\
peticiones de los clientes. \\
Confianza de los clientes en las recomendaciones del personal de \\
contacto, la seguridad de los alimentos y bebidas preparados y \\
capacidad de expresar preguntas sin temor alguno. \\
Capacidad de brindar atención personalizada ante las necesidades \\
específicas de los clientes. \\
Empatía
\end{tabular}

Fuente: Elaboración propia basado de Hernández, et al. (2017), Stevens, Knuston y Patton (1995) y Wolfinbarger y Gilly (2003).

Para representación del modelo propuesto, se tiene la siguiente figura: 
CALIDAD EN EL SERVICIO ELECTRÓNICO EN UNA REPOSTERÍA DE LA CIUDAD DE MÉRIDA, YUCATÁN, MÉXICO.

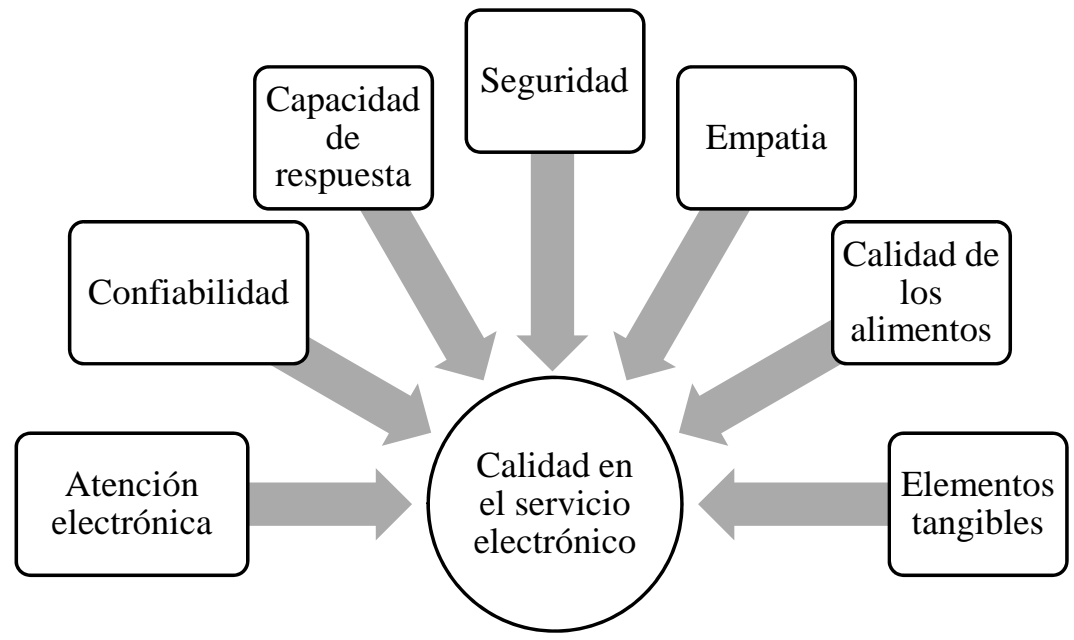

Figura 1. Modelo propuesto para el análisis de la calidad en el servicio.

Fuente: Elaboración propia basado de Hernández, et al. (2017), Stevens, Knuston y Patton (1995) y Wolfinbarger y Gilly (2003).

El objetivo del presente documento es, estimar el nivel de calidad en el servicio electrónico ofrecido por una repostería local a través del modelo adaptado DINESERV, para así determinar áreas de oportunidad y mejora en el establecimiento que permitan mantener su posición en el mercado, así como aumentar la satisfacción del cliente.

\section{MATERIAL Y MÉTODO}

La investigación fue realizada bajo el enfoque cuantitativo, de tipo no experimental, ya que no se manipulan o interviene en las variables de estudio, de alcance descriptivo al centrarse en la identificación de los niveles de calidad de servicio electrónico y de corte transversal porque la información fue recolectada en un solo período, el cual estuvo comprendido entre febrero y marzo de 2020.

\section{Población y muestra}

Para estimar la población de clientes que tenía la repostería se realizó un análisis de los registros de venta de los meses anteriores, resultando un promedio de 55 clientes únicos por mes, por lo que se tomó como base esta cifra para estimar el tamaño de muestra.

La muestra se obtuvo a través de un muestreo aleatorio simple aplicando la fórmula para el cálculo de la muestra para poblaciones finitas contemplando los siguientes parámetros proporcionada por Castañeda, et al. (2002): 
CALIDAD EN EL SERVICIO ELECTRÓNICO EN UNA REPOSTERÍA DE LA CIUDAD DE MÉRIDA, YUCATÁN, MÉXICO.

$$
n=\frac{N Z^{2} p q}{d^{2}(N-1)+Z^{2} p q}
$$

Tabla 2

Elementos para el cálculo de la muestra

\begin{tabular}{ccl}
\hline Elemento & Valor & \multicolumn{1}{c}{ Descripción } \\
\hline Z & 1.96 & $\begin{array}{l}\text { Valor de Z crítico, calculado en las tablas del área de la curva } \\
\text { normal. Llamado también nivel de confianza. En este estudio } \\
\text { fue utilizado un nivel de confianza de } 95 \% .\end{array}$ \\
$\mathrm{D}$ & 0.60 & Error permisible, para este estudio fue utilizado un $6 \%$. \\
$\mathrm{N}$ & 55 & Tamaño de la población. \\
$\mathrm{P}$ & 0.50 & $\begin{array}{l}\text { Probabilidad de éxito de las respuestas afirmativas, se utilizó un } \\
50 \% \text { que permite capturar la mayor variabilidad de los } \\
\text { encuestados. }\end{array}$ \\
\end{tabular}

Fuente: Elaboración propia.

El resultado sugiere que como mínimo deben aplicarse 46 clientes para mantener la representatividad.

\section{Instrumentos}

Para la generación de los datos se elaboró un cuestionario tomando como base el modelo denominado DINESERV, método que puede ser "utilizado por una organización para evaluar y mejorar la calidad en la prestación de servicios" y que fue implementado por Hernandez, Colli, Mex, y Moo (2017), ofreciendo buenos resultados en los niveles de confiabilidad.

Al cuestionario anterior, se decidió adicionar una dimensión más que corresponde a la atención electrónica de tal forma que el instrumento utilizado está conformado por 24 preguntas distribuidas entre las seis dimensiones.

Las dimensiones son ilustradas a continuación: 
CALIDAD EN EL SERVICIO ELECTRÓNICO EN UNA REPOSTERÍA DE LA CIUDAD DE MÉRIDA, YUCATÁN, MÉXICO.

Tabla 3

Estructura del instrumento utilizado

\begin{tabular}{lcc}
\hline Dimensiones & $\begin{array}{c}\text { Cantidad de preguntas } \\
\text { aplicadas por dimensión }\end{array}$ & $\begin{array}{c}\text { Secuencia de número de } \\
\text { preguntas }\end{array}$ \\
\hline Atención electrónica & 3 & $1-3$ \\
Confiabilidad & 4 & $4-7$ \\
Capacidad de respuesta & 3 & $8-10$ \\
Seguridad & 3 & $11-13$ \\
Empatía & 4 & $14-17$ \\
Calidad de alimentos & 3 & $18-20$ \\
Elementos tangibles & 4 & $21-24$ \\
\hline
\end{tabular}

Fuente: Elaboración propia.

Los ítems del instrumento fueron diseñados tomando a consideración las respuestas de percepción, por lo que fue utilizada una escala Likert de cinco niveles, de tal forma que el cliente puede profundizar en su nivel de satisfacción, tal y como se plantea a continuación en forma de tabla las diferentes opciones utilizadas:

Tabla 4

Escala de Likert y puntuaciones utilizadas en el instrumento

\begin{tabular}{ccc}
\hline Nivel de Escala Likert & Significado & Porcentaje \\
\hline 1 & Totalmente en desacuerdo & $0-20$ \\
2 & En desacuerdo & $20-40$ \\
3 & Indiferente & $40-60$ \\
4 & De acuerdo & $60-80$ \\
5 & Totalmente de acuerdo & $80-100$ \\
\hline
\end{tabular}

Fuente: Elaboración propia.

Confiabilidad

Para calcular la confiabilidad del instrumento utilizado para medir la calidad en el servicio en la repostería de estudio, fue utilizado el coeficiente alfa de Cronbach el cual varía de 0 a 1. 
CALIDAD EN EL SERVICIO ELECTRÓNICO EN UNA REPOSTERÍA DE LA CIUDAD DE MÉRIDA, YUCATÁN, MÉXICO.

\section{Tabla 5}

Confiabilidad del instrumento

\begin{tabular}{lc}
\hline Dimensión & Confiabilidad \\
\hline Atención electrónica & 0.86 \\
Confiabilidad & 0.66 \\
Capacidad de respuesta & 0.54 \\
Seguridad & 0.57 \\
Empatía & 0.83 \\
Calidad en los alimentos & 0.83 \\
Elementos tangibles & 0.76 \\
Promedio General & 0.94
\end{tabular}

Fuente: Elaboración propia.

La prueba Alpha de Cronbach genero un resultado global de 0.94, que se interpreta como una alta correlación entre las variables y, por consecuente, una confiabilidad adecuada del instrumento. Este instrumento refiere a, que mientras más cerca se encuentre el resultado de 1, mayor será su fiabilidad. Los resultados obtenidos en la tabla anterior significan una confiabilidad alta en conjunto de todas las dimensiones.

El almacenamiento los datos recopilados fue realizado mediante una hoja de cálculo en Google Sheets ${ }^{\circledR}$.y los cálculos estadísticos fueron efectuados en el software STATA 15®.

\section{RESULTADOS}

Del total de la muestra entrevistada (48 clientes), el $66.66 \%$ (32) de los encuestados son mujeres, mientras que el $43.34 \%$ (16) son hombres, por lo que el sexo femenino fue quien tiene mayor contacto con el establecimiento.

Dentro de los clientes entrevistados, el 48.83\% (22 clientes) estuvieron en el rango de edad de 18 a 25 años, seguidos por grupo de personas entre 26 y 50 años con un $43.75 \%$ (21 clientes) y finalmente un $10.42 \%$ ( 5 clientes) que declararon ser mayores a 50 años. 
CALIDAD EN EL SERVICIO ELECTRÓNICO EN UNA REPOSTERÍA DE LA CIUDAD DE MÉRIDA, YUCATÁN, MÉXICO.

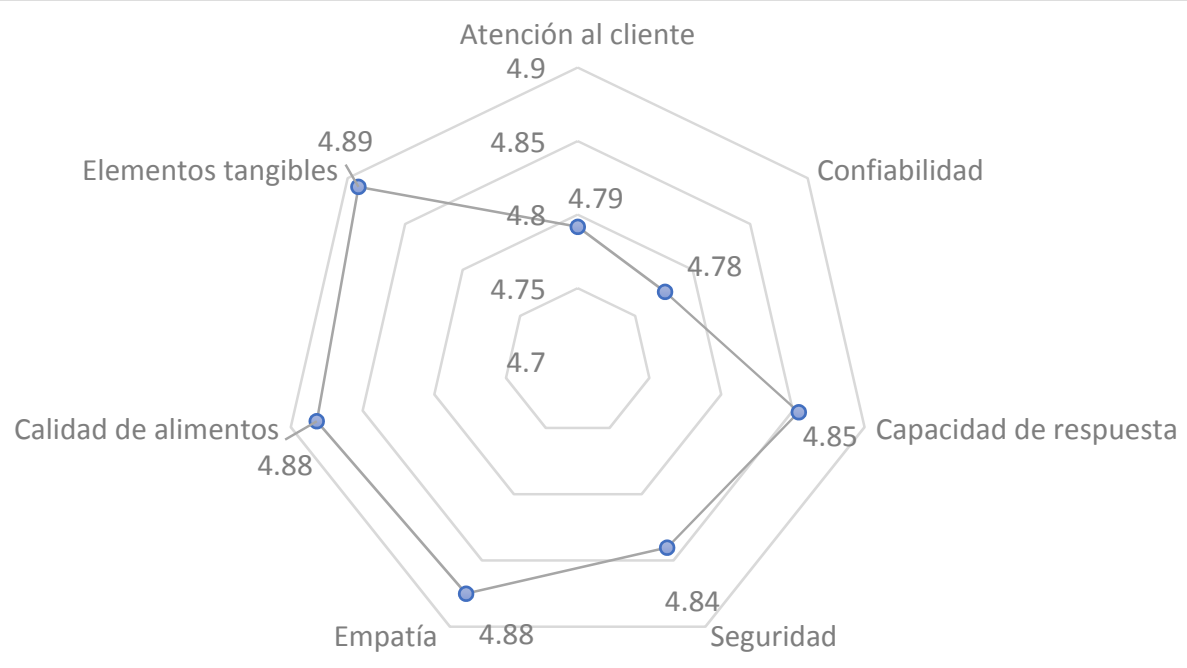

Figura 2. Puntuación promedio por dimensión de calidad en el servicio.

Fuente: Elaboración propia.

De la figura 2, se observa un registro de puntuaciones relativamente elevadas en cada una de las dimensiones, donde la dimensión de "confiabilidad" resultó ser la de menor puntuación (4.78), seguida por la de "atención electrónica al cliente" con 4.79. De acuerdo con los resultados obtenidos, las brechas más importantes se encuentran en que el establecimiento no es tan preciso el proceso de facturación de los postres solicitados, a su vez los tiempos de respuesta mediante solicitudes vía electrónica, no son tan favorables para el entendimiento de los clientes.

Por otro lado, las dimensiones con mayor puntuación son "elementos tangibles" y "calidad de alimentos", con resultados promedio por dimensión respectivamente a 4.89 y 4.88 . Con base en ello es posible decir que ante la situación las áreas a trabajar y de exponer estrategias de mejora son las que menor puntuación poseen, ya que, a pesar de haber obtenido puntuaciones altas, se podrían mejorar pudiendo ofrecer una mejor calidad de servicio al cliente en la repostería. 
Original

CALIDAD EN EL SERVICIO ELECTRÓNICO EN UNA REPOSTERÍA DE LA CIUDAD DE MÉRIDA, YUCATÁN, MÉXICO.

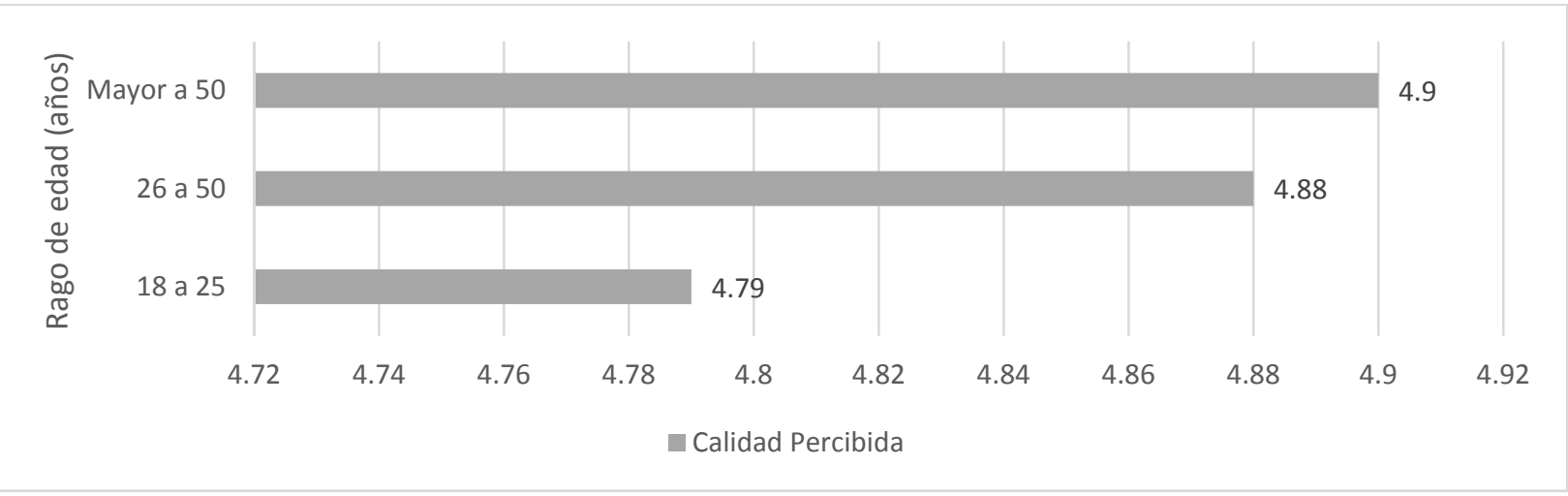

Figura 3. Puntuación promedio por dimensión de calidad y rango de edades.

Fuente: Elaboración propia.

En la figura 4, se ilustran las puntuaciones con el promedio por ítem, facilitando la comprensión de los datos obtenidos y exponiendo la variación existente entre éstos.

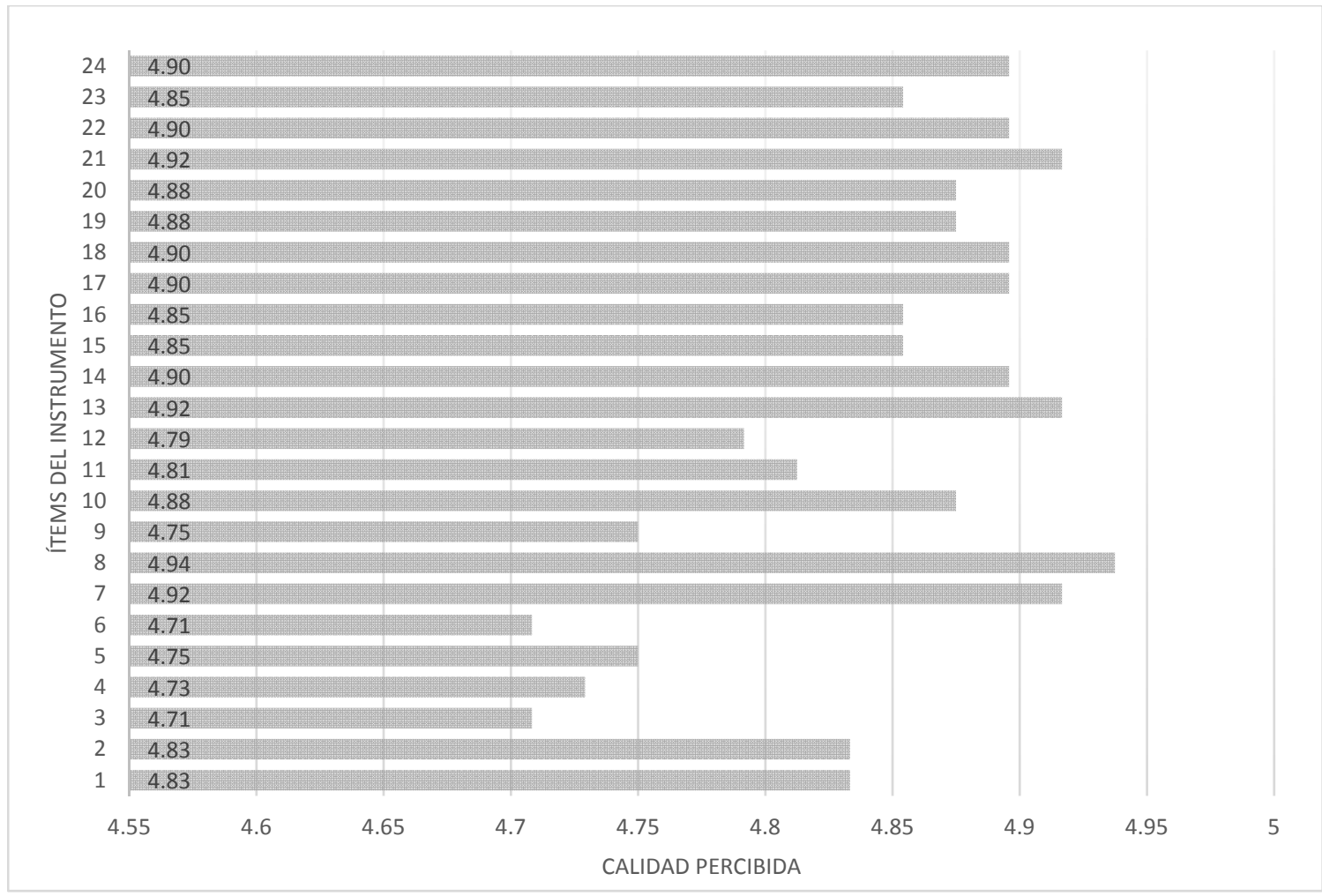

Figura 4. Puntuación promedio por ítem del instrumento.

Fuente: Elaboración propia. 
CALIDAD EN EL SERVICIO ELECTRÓNICO EN UNA REPOSTERÍA DE LA CIUDAD DE MÉRIDA, YUCATÁN, MÉXICO.

\section{CONCLUSIONES}

El objetivo principal de la presente investigación se cumplió, al identificar los niveles de calidad del servicio electrónico percibido por los clientes de la repostería; asimismo, en la mayoría de las dimensiones se obtuvieron resultados que fueron desde lo "de acuerdo" a lo "totalmente de acuerdo", con un promedio general de 4.84 puntos de 5.0 posibles.

A pesar de haber obtenido puntuaciones altas, existen áreas en donde se pueden mejorar, como la correspondiente a la atención electrónica y a la confiabilidad específicamente, donde los clientes percibieron que existe un tanto de problema al tiempo de respuestas o dudas sobre cotizaciones de paquetes o eventos que se requieren a solicitar, así como, a las dificultades de realizar completar transacciones y/o facturaciones dentro de la empresa. Por otra parte, las dimensiones con mayor puntuación fueron: elementos tangibles y calidad de alimentos por lo tanto los clientes se sienten a gusto y satisfechos con el trato que se les brinda al momento de montar el evento solicitado, ofreciendo postres frescos y un servicio especializado, para obtener una satisfacción única del cliente.

Por último, es posible decir que el presente trabajo refleja la utilidad del modelo adaptado del DINESERV para empresas reposteras locales que han comenzado a digitalizar sus servicios, obteniendo niveles de confiabilidad bastante altos. Ante este escenario, es necesario generar mayores instrumentos desde la ciencia administrativa que permitan un mayor grado de profesionalización de las empresas, así como también una ruta para una digitalización más precisa.

\section{REFERENCIAS BIBLIOGRÁFICAS}

Barrera, R., Navarro García, A. y Peris, M. (2014). Evaluación de la calidad en diferentes servicios electrónicos: un análisis multigrupo. Capítulo en XXIV Jornadas Luso Espanholas de Gestao Científica. pp. 1-16.

Bolton, R. N. y Drew, J. H. (1994). Linking customer satisfaction to service operations and outcomes. Service Quality: New directions in theory and practice. Hospitality Research Journal, pp.173-200. 
CALIDAD EN EL SERVICIO ELECTRÓNICO EN UNA REPOSTERÍA DE LA CIUDAD DE MÉRIDA, YUCATÁN, MÉXICO.

Castañeda Jiménez, J., De la Torre Lozano, M., Morán Rodríguez, J. y Lara Ramírez, L. (2002). Metodología de la investigación. México: McGraw-Hill Interamericana Editores.

González, M. y Martínez, D. (2014). Digitalización: su contribución al desarrollo de la gerencia empresarial. Economía y sociedad, 19(45), pp.41-71. .[fecha de Consulta 9 de Mayo de 2020]. EISSN:2215-3403. Disponible en: https://www.revistas.una.ac.cr/index.php/economia/article/view/6042/5950

Guzmán, A. y Cárcamo, M. L. (2014). La evaluación de la calidad en el servicio: caso de estudio "Restaurant Familiar Los Fresnos". Acta Universitaria, 24(3),35-49.[fecha de Consulta 9 de Mayo de 2020]. ISSN: 0188-6266. Disponible en: https://www.redalyc.org/articulo.oa?id=416/41631291004

Hernández, F.I., Colli, N., Mex, F.I. y Moo, C.A. (2017). Calidad en el servicio del comedor de un hospital público medida a través del modelo DINESERV. THEOREMA, p. 139.

Hernández, F.I, Moo, C.A., Cruz Ucán, L.M. y Mex Arriaga. F.I (2019). Análsis de calidad en el servicio a través del modelo DINESERV en una pastelería de Mérida, Yucatán. En VargasDelgado, O. (2019). Collection Ciencias Sociales TI. Editorial Ecorfan. pp.26-37.

Kaynama, S.A. y Black, C.I. (2000): A proposal to assess the service quality of online travel agencies: An exploratory study, Journal of Professional Services Marketing, 21(1), pp. 6388.

Stevens, P., Knutson, B. y Patton, M. (1995). DINESERV: A tool for measuring Service Quality in Restaurants. Cornell Hotel and Restaurant Administration Quarterly, pp.55-59.

Wolfinbarger, M. y Gilly, M. C. (2003): eTailQ: Dimensionalizing, measuring and predicting e-tail quality, Journal of Retailing, 79(3), pp. 183-198.

Zeithaml, V., Parasuraman, A. y Malhotra, A. (2002): Service Quality Delivery Through Web Sites: A Critical Review of Extant Knowledge, Journal of Academy of Marketing Science, 30(4), pp. 362-375. 


\section{CALIDAD EN EL SERVICIO ELECTRÓNICO EN UNA REPOSTERÍA DE LA CIUDAD DE MÉRIDA, YUCATÁN, MÉXICO.}

\section{Anexo. Instrumento}

Le agradecemos se tome el tiempo y nos ayude a contestar la siguiente encuesta, por favor seleccione una respuesta por pregunta, indicando el número que mejor convenga.

Los datos que en ella se consignen se tratarán de manera anónima y empleados únicamente para fines académicos.

Ítems

1. La información proporcionada de los postres es veraz, actualizada y fácil de entender.

2. $\quad$ Los precios de postres (paquetes) requeridos son claros.

3. Fue eficiente la información proporcionada para aclarar dudas.

4. El tiempo de respuesta ante solicitudes vía electrónica (WhatsApp y Messenger) es rápido y preciso.

5. Proporciona al cliente la cuenta/facturación rápida y precisa.

6. Resulta fácil y rápido completar una transacción.

7. Los postres y servicios solicitados son entregados en el tiempo prometido.

8. Los postres y servicios que se venden son acorde a lo prometido.

9. Se ofrece mecanismos de reembolso y/o garantías en el caso de ocurra problemas, son pertinentes.

10. Proporciona un servicio oportuno y rápido.

11. Brinda un esfuerzo extra para manejar sus peticiones especiales.

12. Los empleados pueden responder a sus preguntas de forma completa.

13. El trato brindado lo hace sentir cómodo, confiado y seguro respecto a la preparación higiénica de los postres.

14. Cuenta con personal que está capacitado, competente y experimentado.

15. Los empleados son sensibles a sus necesidades y deseos.

16. Le brindan una atención personalizada haciéndolo sentir especial como cliente.

17. Tiene empleados que son comprensivos y que siempre verifican que todo esté bien.

18. Los postres que se ofrecen son frescos y bien presentados.

19. El sabor de los pasteles y galletas es adecuado con respecto a su temperatura y cocción.

20. El precio de los postres ofrecidos va de acorde a su presentación, tamaño y sabor.

21. La empresa ofrece variedad de opciones de postres.

22. Cuenta con un personal que luce limpio, bien cuidado y propiamente vestido.

23. El montaje (orden y distribución) de los postres es adecuado al tipo de evento solicitado.

24. Los complementos de los postres (bases, vasos y cubiertos) son adecuados para el tipo de evento solicitado.

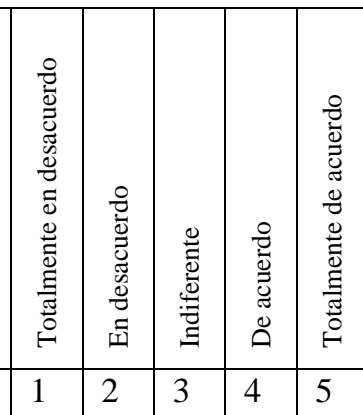

\begin{tabular}{|l|l|l|l|l|}
\hline 1 & 2 & 3 & 4 & 5 \\
\hline 1 & 2 & 3 & 4 & 5 \\
\hline
\end{tabular}

\begin{tabular}{l|l|l|l|l|l|}
1 & 2 & 3 & 4 & 5 \\
\hline 1 & 2 & 3 & 4 & 5
\end{tabular}

